SCIENTIFIC REPORT

\title{
Defining "occludable" angles in population surveys: drainage angle width, peripheral anterior synechiae, and glaucomatous optic neuropathy in east Asian people
}

\author{
P J Foster, T Aung, W P Nolan, D Machin, J Baasanhu, P T Khaw, P-H Alsbirk, P S Lee, S K L Seah, \\ G J Johnson
}

Background/aim: A current consensus in epidemiological studies of primary angle closure (PAC) is to diagnose the condition only if the posterior (usually pigmented) trabecular meshwork is seen for less than $90^{\circ}$ of the angle circumference, termed an "occludable angle." The authors sought to assess the validity of this epidemiological classification by exploring the relation between drainage angle width, peripheral anterior synechiae (PAS) and glaucomatous optic neuropathy (GON).

Methods: 918 Mongolians and 995 Chinese Singaporeans, both groups aged 40 years and older were examined in two population based surveys. Gonioscopic angle width was graded in five categories $(0=$ closed to $4=$ wide open) according the scheme described by Shaffer. Cases with secondary PAS were excluded.

Results: The rate of PAS was between $0.3 \%$ and $1.7 \%$ in people with wide angles (grades 3 and 4). In those with grade 2 angles, PAS were seen in between $8 \%$ of eyes. In eyes with grade 1 angles, the rate rose to $17 \%$ in Chinese Singaporeans, and $31 \%$ in Mongolians. The odds of PAS were higher in people with narrower angles. However, there was a greater absolute number of people with PAS whose drainage angles were classified as "not occludable" than those classified "occludable."

Conclusions: The traditional view that primary angle closure becomes a significant possibility in drainage angles of $\leqslant$ grade 2 (approximately $20^{\circ}$ ) is valid in east Asians. The definition of an "occludable" angle examined here excludes many people with PAS. This probably serves to underemphasise the role of PAC in population surveys of glaucoma prevalence in Asian people.

$\mathrm{P}$ imary angle closure glaucoma (PACG) is a leading cause of blindness in east Asian people. ${ }^{1}$ In epidemiological research, cases of primary glaucoma are usually divided into PACG and primary open angle glaucoma (POAG) on the basis of a subjective judgment on the likelihood of iridotrabecular apposition. A consensus definition of the characteristics an "occludable" drainage angle has come into common usage in epidemiological research. If the posterior (usually pigmented) trabecular meshwork is seen for less than $90^{\circ}$ of angle circumference, this is termed an occludable angle. ${ }^{2-6}$ However, this remains an arbitrary division that has not been validated.

Peripheral anterior synechiae (PAS) are acquired adhesions between the corneoscleral coat and the peripheral iris. They are seen as the hallmark of primary angle closure (PAC). The lesions may vary in height and width. In the earliest stages they are narrow and may extend only as far as the posterior part of the trabecular meshwork in a sawtooth shape. As the process of angle closure progresses, the adhesions extend anteriorly across the trabecular meshwork and spread circumferentially. Typically, PAS, in an intermediate phase of the closure process are multifocal, having a broad base and blunt apex. The extent of PAS correlates with the likelihood of both glaucomatous optic neuropathy, and for laser peripheral iridotomy being deemed insufficient to control the disease. ${ }^{78}$

In this analysis, we sought to investigate the association between width of the drainage angle and the presence of PAS. Furthermore, we were interested to assess the rate of both synechiae and glaucomatous optic neuropathy (GON) in people with and without an "occludable" drainage angle, in order to assess the validity of our assumptions regarding a causal relation between these two clinical entities.

\section{METHODS}

The subjects for this study were recruited during two population surveys of glaucoma prevalence in east Asian people. Ethical approval for these projects was obtained from the Mongolian Ministry of Health and the ethics review board of Singapore National Eye Centre. The work was carried out in accordance with The World Medical Association's Declaration of Helsinki. The methodology of the examination in both Mongolia and Singapore has been described previously. ${ }^{4}$ A summary is given below.

In Hövsgöl province, Mongolia, 1000 subjects aged 40 years and over were chosen from the local government population register, representing $4.8 \%$ of the province's population in this age group. The subjects were drawn from urban (400 people) and rural areas (600 people), using a combination of clustered, random, and systematic sampling.

The electoral register of Tanjong Pagar district in Singapore was used to identify 2000 Chinese subjects from this population (13.3\% of the total), using a disproportionate, stratified, clustered, random sampling procedure. Subjects were drawn randomly, approximately 500 from each of four age strata: 40-49, 50-59, 60-69, and 70-79 years, residing in 50 area clusters defined by street name. The proportion of men and women was determined by the sex ratio of that age group in the district.

Gonioscopy was carried out in all patients using a Goldmann style 2 mirror gonioscope. The examination of

Abbreviations: GHT, glaucoma hemifield test; GON, glaucomatous optic neuropathy; PAC, primary angle closure; PACG, primary angle closure glaucoma; PAS, peripheral anterior synechiae; POAG, primary open angle glaucoma; vCDR, vertical cup:disc ratio 
all subjects was carried out at a low level of ambient illumination throughout. A $1 \mathrm{~mm}$ beam of light was reduced to a very narrow slit. Care was taken to avoid light falling on the pupil during gonioscopy. The assessment was carried out at high magnification $(\times 16$ in Mongolia, $\times 25$ in Singapore). Slight tilting to gain a view over the convexity of the iris was permitted, but further manipulation of the lens or redirection of gaze was avoided because of the possibility of exerting pressure on the cornea and artificially widening the angle.

The width of the angle was estimated in four quadrants as the angle in degrees between a tangent to the surface of the trabecular meshwork and a tangent to the peripheral third of the iris. This was recorded on a five point categorical scale $\left(0^{\circ}\right.$, $10^{\circ}, 20^{\circ}, 30^{\circ}, \geqslant 40^{\circ}$ ) according to the scheme described by Shaffer. 9 The mean angle width for an eye was calculated by adding the angle grade for each quadrant and dividing by 4 . The drainage angle was then graded as occludable or not using a dichotomous modification of the Scheie scheme (based on angle structures seen). ${ }^{10}$ If the posterior (usually pigmented) trabecular meshwork could be seen for less than $90^{\circ}$ of the angle circumference, the angle was classified as "occludable." Dynamic (compression) gonioscopy was used to detect the presence of PAS, unless all angle structures could be clearly identified in a wide open angle. The examination was carried out using either the Goldmann lens or a four mirror gonioscope (Mongolia: Zeiss model, Clement Clark, Harlow, UK, and Singapore: "Sussmann" model, Ocular Instruments, Bellevue, WA, USA). PAS were graded present or absent in four quadrants. Slit lamp examination was used to identify subjects with ocular pathology or surgery that may have caused secondary PAS. These people were excluded from analysis. All gonioscopic examinations were carried out by the same investigator.

Glaucomatous optic neuropathy (GON) was diagnosed if both a statistical abnormality of the vertical cup:disc ratio (vCDR) and a reproducible visual field abnormality were present in the same eye. If a subject's visual function was so severely affected to prevent reliable visual field testing, the diagnosis was made on the grounds of a severely abnormal vCDR. ${ }^{6}$ In Mongolia, the optic disc and posterior pole were examined using a +90D lens through the undilated pupil. If a satisfactory view was not obtained or if an inexplicable field defect was detected the pupil was dilated with $0.5 \%$ tropicamide (Alcon Laboratories, Hemel Hempsted, UK) after gonioscopy, provided the angle was not judged to be occludable. The central visual field was examined using a Henson CFA 3000 (Tinsley Medical Instruments, Croydon, UK). Reproducible field defects (at least two adjacent points, both $5 \mathrm{~dB}$ below age specific threshold) showing a nerve fibre bundle pattern were considered glaucomatous. Equivocal field defects, or those typical of other pathology were classified as non-glaucomatous.

In Singapore, the pupils of all subjects (with the exception of those with established PACG) were dilated with tropicamide $1 \%$ (Alcon-Couvruer, Puurs, Belgium) and phenylephrine hydrochloride $2.5 \%$ (Alcon Laboratories, Fort Worth, TX, USA) drops, repeated twice if necessary. The optic disc was examined at the slit lamp through a fundus contact lens at $\times 40$ magnification. The vertical dimensions of the disc and cup were measured using an eyepiece graticule etched in $0.1 \mathrm{~mm}$ units (Measuring eyepiece, Haag-Streit, Bern, Switzerland). VCDR was calculated as the height of the cup divided by the disc height. Subjects were considered glaucoma suspects on the basis of abnormal screening test results (suprathreshold field screening, occludable drainage angles, raised IOP, and suspect disc morphology). ${ }^{5}$ Glaucoma suspects underwent threshold field testing using the 30-2 pattern of a Mk II Humphrey field analyser (Instrument model 750, Humphrey Instruments, San Leandro, CA, USA).
Tests graded unreliable or compatible with glaucoma were repeated. Tests showing a glaucoma hemifield test (GHT) "outside normal limits" and a reproducible cluster of four contiguous points $(\mathrm{p}<0.5 \%)$ on the pattern deviation plot not crossing the horizontal meridian were considered compatible with glaucoma.

Odds ratios and their confidence intervals, and the confidence intervals of rates, were calculated using the CIA software package (BMJ Publishing Group, London, UK). Logistic regression was used to examine the relation between glaucomatous optic neuropathy and both PAS and occludable angles, while correcting for the effect of age and sex. Logistic regression was carried out using SPSS software (SPSS Inc, Chicago, IL, USA).

\section{RESULTS}

In Mongolia, 942 subjects were examined, representing $94.2 \%$ of those selected within the sampling frame. In Singapore, 2000 names were selected. Of these, 283 were considered ineligible on the grounds that they had either died (46), were severely ill (two), or had moved away from the study area (235). From the remaining 1717 eligible subjects, 1090 people were examined at the research clinic (63.5\%). A further 142 people were examined in their homes, although they underwent a less detailed examination. Data are therefore presented for the 1090 subjects examined in the research clinic.

In Mongolia, complete data on drainage angle width, the presence or absence of PAS in all quadrants, and grading of occludable/non-occludable angle in right eyes were available for 921 of 942 subjects. Two aphakic subjects and one person with evidence of previous severe keratouveitis had PAS in eyes with otherwise wide open angles. These three people were excluded leaving data on 918 subjects for this analysis. When examining the role of PAS in the development of GON, the optic disc status of two subjects could not be determined because of media opacities. These people were excluded leaving 13 with GON and 903 normal subjects.

In Singapore, PAS were identified in $42 \%(=34 / 80)$ of people who had undergone cataract surgery. A further seven people had PAS associated with glaucoma surgery, three subjects had either iris neovascularisation or evidence of significant trauma, and data were incomplete for another five people. These 95 subjects $(80+7+3+5)$ were excluded from analysis, leaving data on 995 subjects $(91.2 \%$ of those examined in the clinic, $57.9 \%$ of all those selected). Of these, 10 had GON and complete data on angle characteristics.

Table 1 gives the age and sex distribution of subjects included in this analysis. Table 2 summarises the rate of PAS according to Shaffer angle width in the right eyes of Mongolians and Singaporeans. The rate of PAS and the odds of PAS increased as angle width decreased.

Table 3 gives the rate of PAS in people with and without an angle graded "occludable." Although there were fewer eyes with PAS and an occludable angle than those with angles "not occludable," the odds of PAS in occludable angles was 21.5 (95\% CI: 9.9 to 46.9) for Mongolian people and 6.6 (95\% CI: 3.3 to 13.4) for Chinese Singaporeans.

Table 4 summarises the relation between PAS and GON. Among Mongolian people, 16.7\% (95\% CI: 7.3 to 33.6) with PAS had GON, compared with $0.9 \%$ (95\% CI: 0.5 to 1.8 ) without PAS. In Singapore, all subjects with PAS and GON had either undergone surgery or were excluded from analysis for another reason. Table 5 gives the rate of GON at various angle widths in Mongolians and Singaporeans. The rates of GON were highest in eyes with the narrowest drainage angles, although the small number of subjects in the Singapore cohort with $10^{\circ}$ angles and glaucoma mean that the confidence intervals overlapped with other groups. 
Table 1 Age and sex distribution of subjects

\begin{tabular}{llllll}
\hline & \multicolumn{2}{l}{ Mongolia } & & \multicolumn{2}{l}{ Singapore } \\
\cline { 2 - 3 } \cline { 5 - 6 } Age (years) & Men & Women & & Men & Women \\
\hline $40-49$ & 159 & 262 & 120 & 139 \\
$50-59$ & 117 & 153 & 106 & 174 \\
$60-69$ & 76 & 81 & 138 & 128 \\
$70+$ & 36 & 530 & 86 & 104 \\
Subtotal & 388 & 530 & 450 & 545 \\
\hline
\end{tabular}

Table 2 Rate of peripheral anterior synechiae according to mean gonioscopic width* in right eyes

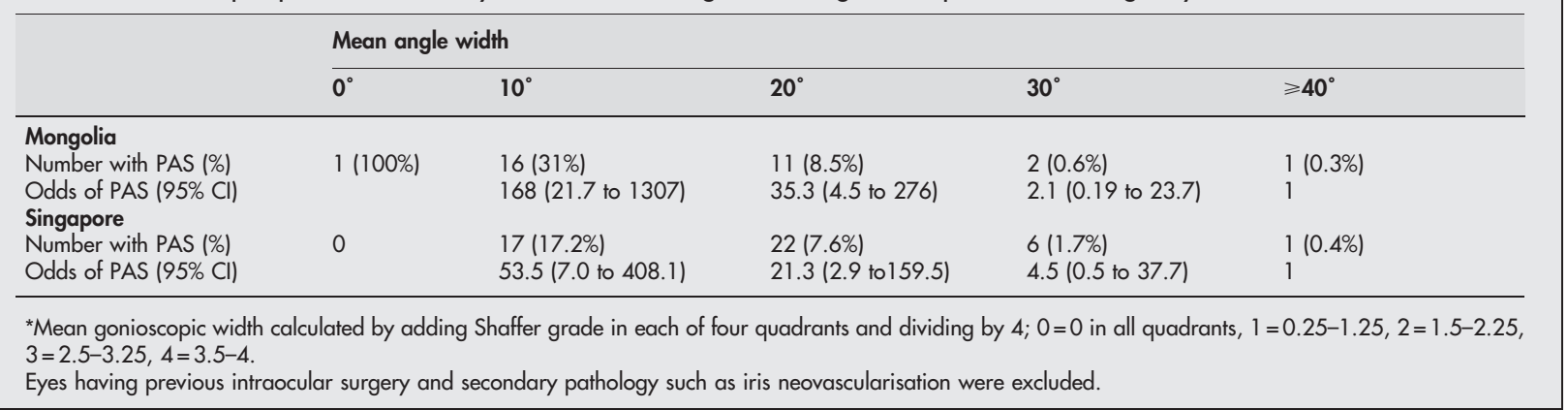

Table 3 Rate of peripheral anterior synechiae in angles graded occludable or not occludable in Mongolian and Singaporean people

\begin{tabular}{|c|c|c|c|c|c|c|}
\hline \multirow[b]{3}{*}{ PAS present } & \multicolumn{4}{|c|}{ Occludable angle } & \multirow[b]{3}{*}{ Total } & \multirow[b]{3}{*}{ OR $(95 \% \mathrm{Cl})$} \\
\hline & \multicolumn{2}{|l|}{ Yes } & \multicolumn{2}{|l|}{ No } & & \\
\hline & Yes & No & Yes & No & & \\
\hline Mongolia & 15 & 37 & 16 & 850 & 918 & (9.9 to 46.9 ) \\
\hline Singapore & 13 & 53 & 33 & 896 & 995 & (3.3 to 13.4 ) \\
\hline
\end{tabular}

Table 4 The rate of peripheral anterior synechiae in people with glaucomatous optic neuropathy in Mongolian and Singaporean people

\begin{tabular}{|c|c|c|c|c|c|c|}
\hline \multirow[b]{3}{*}{ GON present } & \multicolumn{4}{|c|}{ PAS present } & \multirow[b]{3}{*}{ Total } & \multirow[b]{3}{*}{ OR $(95 \% \mathrm{Cl})$} \\
\hline & \multicolumn{2}{|l|}{ Yes } & \multicolumn{2}{|l|}{ No } & & \\
\hline & Yes & No & Yes & No & & \\
\hline Mongolia & 5 & 25 & 8 & 878 & 916 & $22.0(6.7,71.9)$ \\
\hline Singapore & 0 & 46 & 10 & 939 & 995 & $1.1(0.1,19.0)^{*}$ \\
\hline
\end{tabular}

For Mongolian people, multiple logistic regression demonstrated a significant, independent association between greater age $(p=0.002)$, higher IOP $(p=0.004)$, narrower angle width $(p=0.016)$, and GON. Neither the presence of PAS $(p=0.88)$ nor sex $(p=0.75)$ were significant in this model. Repeating this analysis for Chinese Singaporean people, only male sex $(p=0.026)$ and IOP $(p<0.001)$ were significantly associated with GON. We could not detect a significant association between the presence of PAS $(p=0.75)$, angle width $(p=0.73)$, or age $(p=0.19)$ and the presence of GON.

\section{DISCUSSION}

The Shaffer classification scheme requires an examiner to subjectively grade the angle width in degrees, and allocate a grade to one of five categories $\left(0^{\circ}, 10^{\circ}, 20^{\circ}, 30^{\circ}\right.$, and $\left.\geqslant 40^{\circ}\right)$. Angles of $10^{\circ}$ and $20^{\circ}$ are seen to be synonymous with a risk of closure as "probable" and "possible," respectively. ${ }^{9}$ Our data show that this assumption holds true for east Asian populations, where angle closure glaucoma is seen with greater frequency than in European people. ${ }^{45}{ }^{11}$ In eyes with $10^{\circ}$ angles, PAS were seen in one third of Mongolian people and one fifth of Singaporean people. In eyes with $20^{\circ}$ 
Table 5 Rate of glaucomatous optic neuropathy, subdivided according to mean gonioscopic width*

\begin{tabular}{|c|c|c|c|c|c|c|}
\hline & \multicolumn{5}{|l|}{ Mean angle width } & \multirow[b]{2}{*}{ Total } \\
\hline & $0^{\circ}$ & $10^{\circ}$ & $20^{\circ}$ & $30^{\circ}$ & $\geqslant 40^{\circ}$ & \\
\hline \multicolumn{7}{|l|}{ Mongolia } \\
\hline Number with GON & 1 & 6 & 0 & 4 & 2 & \\
\hline \multicolumn{7}{|l|}{ Singapore } \\
\hline Number with GON & 0 & $20 \% 106711$ & 1 & 3 & 4 & 10 \\
\hline$\%$ Rate of GON (95\% CI) & & $2.0 \%(0.6,7.1)$ & $0.3 \%(0.1,1.9)$ & $0.9 \%(0.3$ to 2.5$)$ & $1.5 \%(0.6$ to 3.9$)$ & $1.0 \%(0.5$ to 1.8$)$ \\
\hline $\begin{array}{l}{ }^{*} \text { Mean gonioscopic width } \\
3=2.5-3.25,4=3.5-4 . \\
\text { Eyes having previous intra } \\
\text { The Singapore cohort were } \\
\text { size sampling), and hence }\end{array}$ & $\begin{array}{l}\text { alculated by adding } \\
\text { cular surgery and sec } \\
\text { elected to include pr } \\
\text { he rate of glaucoma }\end{array}$ & $\begin{array}{l}\text { Shaffer grade in each o } \\
\text { condary pathology such } \\
\text { oportionally more elder } \\
\text { does not correspond to }\end{array}$ & $\begin{array}{l}\text { h as iris neovascul } \\
\text { rly subjects than ar } \\
\text { a population pre }\end{array}$ & $\begin{array}{l}\text { dividing by } 4 ; 0=0 \\
\text { sation were exclude } \\
\text { ound in the national } \\
\text { ence. }\end{array}$ & pulation (not using & $\begin{array}{l}25-1.25,2=1.5-2.25 \\
\text { robably proportional to }\end{array}$ \\
\hline
\end{tabular}

drainage angles, about $8 \%$ had PAS. There is a clear "doseresponse" relation between narrower angles and higher rates of PAS. However, it is important to recognise that the absolute number of people with PAS is similar in eyes with $10^{\circ}$ and $20^{\circ}$ angles. In Singapore, more people with PAS had $20^{\circ}$ angles than $10^{\circ}$ angles, although this may reflect the detection, treatment, and thus exclusion from analysis, of people with narrower angles.

PAS are seen as a hallmark of angle closure, although the finding that between $0.3 \%$ and $1.7 \%$ of people with $30^{\circ}$ and $40^{\circ}$ angles had PAS suggests that they can and do occur as a consequence of conditions other than primary angle closure. For example, PAS were seen in $42 \%$ of people who had undergone cataract surgery in Singapore. In phakic people with wide angles we presume that PAS are the consequence of episodes of inflammation, either post-traumatic or idiopathic. The possibility exists that congenital or developmental angle anomalies were misclassified as PAS, although we were conscious of the possibility of confluent bands of iris processes to resemble PAS, if assessed by an inexperienced gonioscopist. It has been noted previously that eyes with dark brown irides may have confluent bands of iris processes extending as far forward as Schwalbe's line. ${ }^{12}$ We were aware of this possibility, and sought to differentiate confluent iris processes from PAS. The fact that all gonioscopic examinations were carried out by a single examiner means that the potential for interobserver variability has been eliminated.

We see $0.5-1 \%$ as the "background" prevalence rate of any PAS in eyes with wide angles $(\geqslant 30)$. In terms of crude prevalence, the rate is 10 times higher in eyes with $20^{\circ}$ angles, and 20-30 times higher in $10^{\circ}$ angles. It is recognised that angle width decreases with increasing age. Any more thorough analysis must therefore consider the effect of age. Multiple logistic regression identified no significant association between age and PAS, once angle width had been included in the analysis. It appears that, in terms of prevalence of PAS in a population, significant angle closure begins in some eyes with $20^{\circ}$ angles.

The designation of an "occludable" angle has been used in several epidemiological studies to divide subjects in to categories of primary open angle glaucoma (POAG) and primary angle closure glaucoma (PACG)..$^{2-5}$ People with $20^{\circ}$ angles, and some with $10^{\circ}$ angles, will not meet the diagnostic criteria for an "occludable" angle. It is troubling that under the current classification scheme, at least half those who are experiencing angle closure (as indicated by PAS) and have glaucomatous optic neuropathy would not be included as cases of PACG. This suggests that definition of an occludable angle in widespread use may be too stringent. It is important that people with POAG and narrow but open drainage angles are not classified as cases of PACG. Hyams et al found that six of 267 eyes undergoing iridotomy for presumed primary angle closure glaucoma had "mixed mechanism" disease $(2.2 \%)$, and a further three were felt to have POAG with narrow angles $(1.1 \%) .{ }^{13}$ If one assumes a background prevalence of PAS of $1 \%$, and POAG of $2 \%$ in an adult population, one would expect a chance association of the two findings to occur in two in 10000 people, if angle width were not brought into consideration. Currently, the potential for significant misclassification seems unduly prejudiced against diagnosis of PACG.

In this study we have used a figure for mean angle width derived from all four quadrants. Variation in angle width between quadrants is not reflected using this method, and introduces some lack of precision in estimating the relation between PAS and gonioscopic anatomy. However, we did not find PAS to be consistently present only in the narrowest quadrants. Furthermore, our analysis gives equal weight to eyes with limited and extensive areas of PAS. These issues may have hindered our ability to examine the true relation between the factors in this study.

We recognise that the small numbers of subjects with GON were small ( 10 cases in Singapore and 13 cases in Mongolia). Among the Singaporean subjects especially, it was necessary to exclude of a large number of previously diagnosed and surgically treated cases. In our original prevalence report from Singapore, we reported 45 cases of glaucoma in the 1232 subjects examined in all phases of the survey. ${ }^{5}$ Among these, two of 22 cases of POAG had been diagnosed, both of whom had been operated on. There were 10 of 14 cases of PACG previously diagnosed, all of whom had been operated on in their right eye, and therefore excluded. ${ }^{5}$ The characteristics of the angle closure process has been altered in Singaporean subjects who have sought medical attention, making it impossible to examine the role of untreated angle closure in the development of visually significant glaucomatous optic neuropathy. The data on Mongolians give a clearer cross sectional view of untreated PAC and PACG in an entire population. However, the consistency of the trends we have detected suggests that, in terms of the broad concepts, the data are mutually supportive.

In conclusion, if PAS do indicate that functionally significant angle closure is occurring, then these data suggest that the most widely used epidemiological definition of an "occludable" angle is too stringent. This will lead to misclassification of many subjects with PACG as cases of POAG. There is undoubtedly no substitute for detailed static and dynamic gonioscopy by an experienced ophthalmologist when considering a diagnosis of PACG, and if possible, this should remain the diagnostic "gold standard." We believe that the evidence presented here suggest that the definition 
of an "occludable angle" used by our group and others in epidemiological studies of glaucoma should be reconsidered.

\section{ACKNOWLEDGEMENTS}

Survey work in Singapore was funded by the National Medical Research Council, Singapore, through a grant to Singapore Eye Research Institute (SERI). The British Council for the Prevention of Blindness and The International Glaucoma Association, London, supported the survey in Mongolia.

This work was presented in abstract at the 2001 annual meeting of the Association for Research in Vision and Ophthalmology, Fort Lauderdale, FL, USA.

The authors thank Dr Paul Healey for raising this research question.

\section{Authors' affiliations}

P J Foster, T Aung, S K L Seah, Singapore National Eye Centre and Singapore Eye Research Institute, Singapore

P J Foster, T Aung, W P Nolan, P T Khaw, P S Lee, G J Johnson, The Institute of Ophthalmology, University College London, UK

P J Foster, T Aung, W P Nolan, P T Khaw, Glaucoma Research Unit, Moorfields Eye Hospital, London, UK

D Machin, Division of Clinical Trials and Epidemiological Science,

National Cancer Centre, Singapore

J Baasanhu, Central Medical University Hospital, Ulaanbaatar, Mongolia

P-H Alsbirk, Department of Ophthalmology, Hillerød Hospital, Denmark

Correspondence to: P J Foster, Division of Epidemiology, Institute of Ophthalmology, Bath Street, London ECIV 9EL, UK; p.foster@ucl.ac.uk
Accepted for publication 10 June 2003

\section{REFERENCES}

1 Foster PJ, Johnson GJ. Glaucoma in China: how big is the problem? Br J Ophthalmol 2001;85:1277-82.

2 Arkell SM, Lightman DA, Sommer A, et al. The prevalence of glaucoma among eskimos of Northwest Alaska. Arch Ophthalmo 1987; 105:482-5.

3 Salmon JF, Mermoud A, lvey A, et al. The prevalence of primary angle-closure glaucoma and open angle glaucoma in Mamre, Western Cape, South Africa. Arch Ophthalmol 1993;111:1263-9.

4 Foster PJ, Baasanhu J, Alsbirk PH, et al. Glaucoma in Mongolia-a population-based survey in Hövsgöl Province, Northern Mongolia. Arch Ophthalmol 1996;114:1235-41.

5 Foster PJ, Oen FT, Machin DS, et al. The prevalence of glaucoma in Chinese residents of Singapore. A cross-sectional population survey in Tanjong Pagar district. Arch Ophthalmol 2000;118:1105-11.

6 Foster PJ, Buhrmann RR, Quigley HA, et al. The definition and classification of glaucoma in prevalence surveys. Br J Ophthalmol 2002;86:238-42.

7 Salmon JF. Long-term intraocular pressure control after Nd-YAG laser iridotomy in chronic angle-closure glaucoma. J Glaucoma 1993;2:291-6.

8 Nolan WP, Foster PJ, Devereux JG, et al. YAG laser iridotomy treatment for primary angle-closure in east Asian eyes. Br J Ophthalmol 2000;84:1255-9.

9 Becker B, Shaffer RN. Diagnosis and therapy of the glaucomas. St Louis: CV Mosby, 1965.

10 Scheie HG. Width and pigmentation of the angle of the anterior chamber. A system of grading by gonioscopy. Arch Ophthalmol 1957;58:510-12.

11 Seah SKL, Foster PJ, Chew PT, et al. Incidence of acute primary angle-closure glaucoma in Singapore. an island-wide survey. Arch Ophthalmol 1997; 115: 1436-40.

12 Lichter PR. Iris processes in 340 eyes. Am J Ophthalmol 1969;68:872-8.

13 Hyams SW, Keroub C, Pokotilo E. Mixed glaucoma. Br J Ophthalmol 1977;61:105-6. 